\title{
A Survey on Tools Essential for Semantic Web Research
}

\author{
Jenice Aroma $\mathrm{R}$ \\ Post Graduation Student \\ Karunya University \\ Coimbatore
}

\author{
Mathew Kurian \\ Assistant Professor \\ Karunya University \\ Coimbatore
}

\begin{abstract}
The Syntactic web search scheme is upgraded by adding semantics through the recent trends of Semantic web. The importance of Semantic web is due to the increased level of appropriate results on information retrieval. It brings a wide participation of researchers all over the world. Even beginners could gain an easier understanding of the Semantic web standards. This paper clearly illustrates some of the tools and technologies that are applied for effective semantic web development. Most of the efficient tools are open for access. Certain commercial tools for specific mode of development are also available.
\end{abstract}

\section{Keywords}

Reasoner, Semantic web, WSDL.

\section{INTRODUCTION}

The web resources are growing day by day, on addition of new information. As the size of information reserved gets increased, the need for efficient search scheme to retrieve the results more relevant also increased. In syntactic based discovery, the relevant pages are retrieved as required results. But, it doesn't satisfy the expected outcome of results. Hence, an efficient mechanism of providing the expected results is a need. The Syntactic discovery of web pages is restricted to keywords, due to the underlying HTML web pages. These HTML pages favour easier human reading. But, It doesn't improve the quality in retrieval of results. It can be resolved through structuring the existing web content through addition of Semantics. Thus, Semantic web is formulated.

The HTML can describe only the documents and their links among them. In Semantic web, certain technologies like Resource Description Framework (RDF), Web Ontology Language (OWL) and Extensible Markup Language (XML) are combined in order to replace the existing web content. These technologies provide machine readable descriptions that enable structuring of web content through addition of meaning. Thereby, it brings out an efficient search scheme with automated information processing.

To enable this structuring of existing web content, an automated mapping scheme is a need. The recent W3C standard like Web Ontology Language for Service (OWL-S) favours this automation of web services. This mode of automation leads to Semantic Mapping of concepts. To build this semantic web into practice, certain tools are in need to be known for the better development. This paper gives a helping hand to beginners in this field of research to have a glimpse of tools that are in need to be known.

The following sections describe the purpose of these tools in Semantic building of web:

\subsection{Ontology Editor}

The Ontology Editors are used for creating a data model for the underlying Semantic web Application. It favours
The Semantic Mapping of concepts be detailed with the following sections of this paper. The main contribution of this survey on Semantic web tools is to give a clear overview of Tools and their purpose in building Semantic web. The Semantic Web researchers all over the world bring out different ideas, and tools and technologies needed for Semantic web are open to access. It is a primary need for web today with information overload, to meet Semantic web in true.

\section{SEMANTIC MAPPING}

The Semantic web is otherwise termed for web of Data. The main goal of Semantic web is to achieve machine interoperability. The current web services are based on Web Service Definition Language (WSDL). It is a XML format that describes services as a set of end points operating on messages, containing either document oriented or procedure oriented information. Normally, a service is a container for a set of system functions that are exposed to web based protocols. Similarly in Web services, a client reads WSDL file to determine the operations available on the Server. Then SOAP is used to call one of those operations listed in WSDL.

On Semantic Mapping, these WSDL services are mapped to OWL-S services. It can be done through the addition of semantic annotations. It is a tedious task to be done manually. The automation of semantic mapping is possible with OWL-S services. The prior semantic mapping approaches using Darpa Agent Markup Language (DAML) are semi automated. Many Semantic mapping schemes that are based on OWL-S Services are available with complete automation [4].

\section{TOOLS FOR SEMANTIC WEB}

In this section, the tools that are more essential for building a semantic web are detailed. The upgrade of Semantic web brings many easy-to-use semantic tools, which are available for open access. Many Commercial tools are also available for building Semantic web Applications.

The Semantic web tools can be grouped according to their purpose [1]. The following are three categories of Semantic web tools. First, the ontology Editors are used for data modeling. The visualization and editing of Ontologies are added to be the extensions of Ontology Editors. Second, the Reasoner for the notion of generalized Inference mechanism. Finally, the Plugin and APIs that can set up the Semantic web development environment.

This paper details some of the tools under these categories in the later sections of this paper. It offers a brief overview of their features and purpose in building Semantic web.

manipulating the available ontologies for access. The ontologies are constructed using standard Ontology languages. The Ontology Languages are formal languages that depends with reasoning rules for framing these ontologies [3]. 
The Ontology Languages are classified either by syntax or structure. The Syntax oriented Ontology Languages like OWL, Resource Description Framework (RDF), Ontology Inference Layer (OIL) are more prevalent ontology languages [9]. The Ontology Editors can be classified based on the type of ontology languages used.

Protégé, SWeDe are some of the freely available Ontology editors, where commercial ontology editors like TopBraid Composer also available. Limited features are only available with the free editions of these commercial tools.

\subsection{Reasoner}

The Inference mechanism on Reasoners are the rich capability provided to infer logical consequences from axioms. These Logical consequences are nothing but the relationship between statements that are true, where axioms are the statements which are accepted to be true without controversy

OntoBroker, DLog and RacerPro are some of the commercial Reasoners [2]. The freely available Reasoners like Pellet, FaCT++, HermiT are applicable to support the semantic web applications.

The Pellet reasoner which is more prevalent to be used nowadays is detailed in the later sections of this paper

\subsection{Plugin and API}

The Application Programming Interface (API) is a set of protocols, and tools for building software applications. It favour easier development of a program by providing necessary building blocks and assembling these building blocks is the remaining part to work,[5]. The APIs are more useful for users than programmers. Since, they provide a common API with similar interface for all programs. This leads to an easier learning task for even naïve users.

The plug-ins are a set of software components used for adding specific abilities to a larger software application. For Example, Pellet Reasoner Plugins are added in Eclipse IDE for inferring the relationships. Similarly plug-ins like Adobe Flash Player and Java Applets are commonly used in web browsers to play video and scan for viruses.

\subsection{Other Tools}

The Query building tools like SPARQL and ARQ Engine etcdepends with their underlying Semantic web query languages like SPARQL Protocol and RDF Query Language (SPARQL). These query languages are used to manipulate and retrieve the content from RDF Descriptions.

\section{POPULAR SEMANTIC WEB TOOLS}

The following section details the most prevalent semantic web tools that can be easily applied with the semantic web development. In Ontology editors, the purpose of Protégé be shown in brief. The features of Jena API and Reasoners like Pellet, FaCT and Hermit are also detailed.

\subsection{Protégé}

Protégé is being developed at Stanford University in collaboration with the University of Manchester. It is a free, open-source platform that provides wide range of tools to construct domain models and knowledge-based applications with ontologies. Protégé can also be used as either plugins or API for building applications.
There are two ways of modeling ontologies: The Protégé Frames editor is used to build and populate ontologies that are frame-based on considering Open Knowledge Base Connectivity protocol (OKBC). The Protégé-OWL editor is used to build ontologies for Semantic web using W3C's Web Ontology Language (OWL). The purpose of Protégé-OWL editor be detailed below.

The Protégé-OWL editor is designed to be an extension that supports the Web Ontology Language (OWL). An OWL ontology may include descriptions of classes, properties and their instances [6]. The OWL-S is the most recent standard of ontology languages developed World Wide Web Consortium (W3C) to bring out Semantic web.

The Protégé-OWL editor enables users to: Loading ontologies, editing classes and properties, defining OWL expressions and using reasoners. It is easy to configure with extended adaptability to different development environments. It has an open-source Java API for development of web services.

\subsection{SWeDE}

The SWeDE (Semantic Web Development Environment) toolkit, provides an OWL editor with essential features like syntax highlighting, auto completion, and error-detection. It works with Eclipse IDE. It combines working of many existing tools, even the prominent tool like OWL Validator. In further, SWeDE will integrate more visualization and editing tools for Semantic Web documents and rules [7]. It provides extended RDF models and visual editors.

\subsection{Jena API}

The Jena API is a well known Semantic web platform among Java framework for building Semantic Web applications. It includes wide range of java libraries that help developers to develop code easier. The very first approach of developing Jena was underwent by researchers in HP Labs by 2000 . Jena is an open-source project, and has been used in a wide variety of semantic web applications. In 2010, Jena was adopted by the Apache Software Foundation.

The distinguished feature of Jena is to perform reasoning based on OWL and RDFS ontologies using a very appropriate inference mechanism that depends with the inference engine is provided [8].The features of Jena Framework includes reading, processing and writing as being an API. Pellet

\section{Reasoner}

Pellet is a complete OWL-DL reasoner which offers effective reasoning and debugging of ontologies. It is open source and freely available for the researchers to apply the inferring ability in their Semantic web applications. The extension to Pellet Reasoner includes Reasoners for OWL-DL ontologies. It extends support for hybrid reasoning using rules,[12].

Pellet provides easier access for even naïve users through incorporating various interfaces. The command line interface is one among those interfaces. It offers inferring through conjunctive query answering, rule support and axiom pinpointing. 
In addition, Pellet improves the reasoning performance using new optimizations in the presence of enumerated classes and individuals.

\subsection{HermiT Reasoner}

HermiT is a new OWL reasoner. It depends with "hypertableau" calculus. It resolves the issues on nondetermnistic approaches which degrade the performance. The advanced feature of reusability than generating new ones achieve better optimization. Moreover, many other approaches of ontology classification and nominal handling are also optimized [10].

The special feature of HermiT Reasoner is the classification of Ontologies. The prior Reasoners are also capable with ontology classification through depicting the number of ontologies. But, HermiT remains more efficient than other reasoners in Ontology Classification.

\subsection{Fact ++ Reasoner}

The FaCT++ is a DL reasoner (Description Logics) where the prior reasoners shown above are a combination of OWL reasoner. It acts as a better platform which is adaptable to new tableaux algorithms and optimization techniques, other than the techniques with FACT system. It leads to further enhancements towards Heuristic Optimizations.

The Description Logics are logic based knowledge representations of data. OIL, DAML+OIL and OW are the most important ontology languages that are applied with these Description Logics. These DLs are added to the ontology languages for addition of computational services. This extension of ontology languages favour their use in different applications,[11].

The reasoners are used for inferring the capability of ontologies. The Inference mechanisms in reasoners are applied for bringing out a better manipulation of complex For example: The use of ontologies in building applications are increasing day by day. It leads to an increased level of complexity to manipulate the ontologies. Hence, Reasoners can be used to analyze the issues on the performance due to huge size of ontologies.

\subsection{Comparison of Semantic web tools}

The following table TABLE I illustrates the features of various Semantic web tools that are available in open source for building Semantic web applications.

TABLE I - LIST OF SEMANTIC WEB TOOLS.

\begin{tabular}{|c|c|l|}
\hline Type & Tools & \multicolumn{1}{c|}{ Features } \\
\hline $\begin{array}{c}\text { Ontology } \\
\text { Builder }\end{array}$ & Protégé & $\begin{array}{l}\text { Data modeling of } \\
\text { ontologies. }\end{array}$ \\
\cline { 2 - 3 } & SWeDE & $\begin{array}{l}\text { Integrated visualization } \\
\text { of Ontologies. }\end{array}$ \\
\hline $\begin{array}{c}\text { Application } \\
\begin{array}{c}\text { Programming } \\
\text { Interface } \\
\text { (API) }\end{array}\end{array}$ & Jena API & $\begin{array}{l}\text { An Integrated semantic } \\
\text { web platform for easier } \\
\text { Semantic web building. }\end{array}$ \\
\hline
\end{tabular}

\begin{tabular}{|c|c|c|}
\hline \multirow{3}{*}{ Reasoners } & Pellet & OWL-DL Reasoner \\
\cline { 2 - 3 } & HermiT & OWL Reasoner \\
\cline { 2 - 3 } & FactT & DL Reasoner \\
\hline
\end{tabular}

\section{OTHER COMMERCIAL TOOLS}

The Commercial Tools offer an extended support than the tools available that are available in open source. The Commercial tools are owned by their vendors and trial editions are available only with limited features. The following section details the features of two more prevalent Commercial tools like TopBraid Composer and RacerPro.

\subsection{TopBraid Composer}

The TopBraid Composer provides a suitable environment for the user to develop the Semantic web applications easily. It offers an efficient modeling environment for developing Semantic Web ontologies. It supports developing ontologies, managing data model and testing configurations of data models. TopBraid Composer has the combination of both RDF editor and OWL ontology editor. It can also be applied as the SPARQL tool for querying in Semantic web applications.

This tool can also be available as free edition with limited features. But the complete enterprise version be available in Maestro and Standard edition. The Maestro edition be used for processing data chains and developing integrated data services through using SPARQL MotionScripts. It has a own internal web server for testing applications, which leads to significantly improved turn-around times in application development.

In Standard edition of this tool, It provides visual editors for developing RDF graphs and class diagrams. It has scalable database backends. It also provides automated conversion of XML, XSD, Excel, UML and other data sources. The Standard Edition can connect to relational databases to run SPARQL queries over relational data.

It provides refactoring support and it has a set of features that supports the entire life cycle of a Semantic application development. It can also be used as a run-time environment to execute rules, reasoners and queries.

\subsection{RacerPro}

The RacerPro is used as a reasoning engine for Ontology editors like Protégé. It can also be termed for an information repository in Semantic web with optimized retrieval engine to handle large sets of data. It manages the Semantic web ontologies based on OWL. The term RACER in RacerPro stands for Renamed ABox and Concept Expression Reasoner.

The Description logics that provide the foundation of international approaches to standardize ontology languages in the context of the so-called semantic web be the origin of RacerPro.

The RacerPro is used for checking the consistency of an OWL based ontology and a set of data descriptions. It is used to find the subclass relationships in the ontology declaration. It includes a HTTP client for retrieving imported resources from 
the web. It allows importing multiple resources into a single ontology.

\subsection{Comparison on features of Free and Commercial Semantic Web Tools}

This section contributes a brief overview on the features of two most popular Ontology Builders. The Comparison focuses on bringing out the essential features of both freely available and Commercial Tools of Ontology modeling. Protégé is the most popular Ontology Builder and TopBraid Composer is a commercial tool that has free editions also. The extended feature of the commercial edition is discussed for comparison. The following TABLE II illustrates this overview on features of Ontology Builders.

TABLE II - PROTÉGÉ VS TOPBRAID COMPOSER

\begin{tabular}{|c|c|c|}
\hline Features & Protégé & $\begin{array}{c}\text { TopBraid } \\
\text { Composer }\end{array}$ \\
\hline Developers & $\begin{array}{c}\text { SMI Stanford } \\
\text { University }\end{array}$ & TopQuadrant \\
\hline Availability & Open Source & Software License \\
\hline Extensibility & Plug-Ins & Plug-Ins \\
\hline $\begin{array}{c}\text { Support for other } \\
\text { Ontology Tools }\end{array}$ & FaCT and Jena & Sesame and Jena \\
\hline $\begin{array}{c}\text { Inference Engine } \\
\text { Support }\end{array}$ & Available & WOL, SPARQL \\
\hline $\begin{array}{c}\text { Other Inference } \\
\text { Engine }\end{array}$ & $\begin{array}{c}\text { RACER, FaCT, } \\
\text { FaCT++, Pellet }\end{array}$ & $\begin{array}{c}\text { Pellet and } \\
\text { SPARQL }\end{array}$ \\
\hline $\begin{array}{c}\text { Exception } \\
\text { Handling }\end{array}$ & Not Available & Available \\
\hline
\end{tabular}

\section{CONCLUSION}

This paper shows a brief overview of tools that are applied with semantic web applications. The Semantic web is a major need for this world today that depends on web for information. Even a single user among the millions of users in web holds a huge data to handle and adding new data to be stored every day. It leads to a necessity of bringing out an efficient search scheme that resolves all the issues on information retrieval with syntactic discovery.

The need for efficient scheme of information retrieval leads to the need for Semantic web. And it bring researchers all over the world to focus on this dream of Tim Berners Lee, Semantic web (web 3.0) to come true. The goal of this paper is to offer a better understanding of the variety of Semantic web tools to researchers and even newbie to this semantic web research.

\section{REFERENCES}

[1] Karin K.Breitman, Marco Antonio, Walter Truszkowski 2007 Semantic web: Concepts, Technologies and applications, NASA Monographs in systems and software Engineering.Springer.

[2] R.B Mishra, Sandeep kumar, 2010. Semantic web reasoners and languages.Springer Online Edition.

[3] Sabin Corneliu Buraga, Liliana Cojocaru, Ovidiu Cătălin Nichifor 2006. Survey on Web Ontology Editing tools.Transactions on Automatic control and computer science.

[4] Jenice Aroma R, Mathew Kurian, 2012. A Survey on need for Semantic web. International Journal of Scientific Research and Publications.

[5] Jim Hendler, Mike Dean, 2005. Semantic web Tools Tutorial. Semantic web Applications for National Security.

[6] Protégé [online]: http://protege.stanford.edu/

[7] SWeDe - The Semantic Web Development Environment [online]:http://owl-eclipse.projects.semwebcentral.org/

[8] Jena API [online]:http://www.hpl.hp.com/semweb/

[9] Ontology Editor - The list of Ontology Editors available. [online]: http://en.wikipedia.org/wiki/Ontology_editor

[10] Rob Shearer, Boris Motik, and Ian Horrocks. HermiT: A Highly-Efficient OWL Reasoner.

[11] Dmitry Tsarkov and Ian Horrocks. Fact++ Description Logic Reasoner: System Description.

[12] Evren Sirin, Bijan Parsia , Bernardo Cuenca Grau, Aditya Kalyanpur, Yarden Katz. Pellet: A Practical OWL-DL Reasoner. 